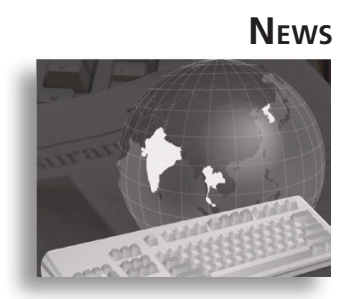

Research Highlights

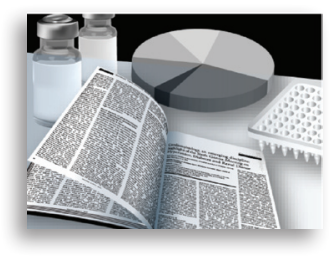

\section{Long-lasting immunity induced by synthetic nanoparticles incorporating multiple adjuvants}

Researchers at Emory Vaccine Center (GA, USA) have recently demonstrated that nanoparticles designed to resemble viruses and mimic the immunostimulating effect of the yellow fever vaccine (YF-17D) are able to include lifelong immunity in mice.

The nanoparticle is composed of biodegradable polymers and is similar in size and immune composition to viruses. The researchers were led by senior author Bali Pulendran at Emory University School of Medicine, and aimed to develop a particle able to induce an immune response similar to the long-lasting and protective response induced by YF-17D. The yellow fever vaccine is capable of protecting against diseasecausing forms of the virus for decades and previous investigation by Pulendran and his group into the mechanism of action of YF-17D revealed that the innate immune system is capable of sensing the vaccine via multiple Toll-like receptors (TLRs) located on dendritic cells.

In addition to antigen, the team incorporated two adjuvants into the poly(lactic acid)co-(glycolic acid) particles, both of which are US FDA approved for use in humans individually: monophosphoryl lipid A, a low-toxicity derivative of the TLR4 ligand lipopolysaccharide; and imiquimod, which mimics the effects of viral RNA and targets TLR7. They found that immunization with these nanoparticles induced increases in antigen-specific neutralizing antibodies in league with those produced in response to immunization with nanoparticles containing antigens and a single TLR ligand.

The group also noted an enhanced persistence of germinal centers and of plasma-cell responses within the lymph node that persisted for $>1.5$ years. This ability to induce a long-lasting immune response could benefit future vaccine design.
"Most of the highly successful vaccines such as live-attenuated vaccines against smallpox, yellow fever, measles, mumps and rubella, induce immunological memory that can last a lifetime. The ability to do this using a synthetic nanoparticle could facilitate the design of safe vaccines against a host of diseases such as malaria, HIV and TB. Furthermore, these nanoparticle-based vaccines might offer a strategy to prolong the duration of immunity stimulated by vaccines that typically do not induce long-lived responses, such as the carbohydrate vaccines against meningococcal and pneumococcal diseases, as well as the pertussis vaccine," explained Pulendran.

In addition to inducing antibody-producing plasma cells, the nanoparticles were also able to induce an effective T-cell response.

"The magnitude of the antigen-specific CD8 ${ }^{+} \mathrm{T}$-cell response was as high as that observed with viral infections," said Pulendran. "The $\mathrm{CD}^{+}$T-cell response against Bacillus anthracis protective antigen lasted as long as 1.5 years.

"The next step is to test the immunogenicity of these particles in nonhuman primates, using a variety of antigens, including antigens from HIV, malaria and dengue. We hope that this platform would represent a universal adjuvant platform that could be coupled with any antigen. However, the feasibility of using this in humans, must await several nonhuman primate studies (which are currently being performed), as well as a safety trial in humans."

Sources: Kasturi SP, Skountzou l, Albrecht RA et al.: Programming the magnitude and persistence of antibody responses with innate immunity. Nature 470(7335), 543-547 (2011); Emory University press release: $h$ ttp://shared.web.emory.edu/whsc/ news/releases/2011/02/virus-mimicking-nanoparticles-can-stimulate-long-lasting-immunity.htm/ 
In the new [GlaxoSmithKline]-Abbott agreement, a PCR-based test will be developed to detect MAGE-A3 in melanoma tumor biopsies.

\section{Abbott and GlaxoSmithKline to} develop test for selecting patients for skin cancer immunotherapy

Abbott has recently announced that it has formed an alliance with GlaxoSmithKline (GSK) to develop a molecular diagnostic test intended for use as an aid in selecting patients who may benefit from a particular skin cancer treatment.

Melanoma is the most deadly form of skin cancer. When diagnosed early, it is nearly $100 \%$ curable; however, distant metastasis is generally considered incurable with a 5-year survival rate of less than $10 \%$.

GSK has developed a MAGE-A3 antigenspecific cancer immunotherapeutic (ASCI) that is currently being evaluated in melanoma biopsy specimens in Phase II clinical trials. This is one of a novel class of ASCI medicines that combine tumor antigens, delivered as purified recombinant proteins, and immunostimulants designed to increase the antitumor immune response. The combination is intended to train the immune system to recognize and eliminate cancer cells in a highly specific manner. The specificity of ASCI means that it is only suitable for patients with melanoma tumors expressing the tumor-specific antigen, MAGE-A3.

In the new GSK-Abbott agreement, a PCR-based test will be developed to detect MAGE-A3 in melanoma tumor biopsies. The test will be designed for use on the Abbott $\mathrm{m} 2000^{\mathrm{TM}}$ automated molecular instrument system comprising the m2000sp for automated sample preparation and the m2000rt for real-time PCR detection and analysis.

If approved by the FDA, this would be the first nucleic acid-based test for use in identifying patients for targeted skin cancer therapies.

Source: Abbott press release: www.abbott. com/global/url/pressRelease/en_US/Press_ Release_0827.htm

\section{Isconova aim to optimize West Nile virus vaccine with Matrix $\mathrm{M}^{\mathrm{TM}}$ adjuvant}

Isconova (Uppsala, Sweden) has recently been selected in a new EU project, the objective of which is to develop a new vaccine against the viral disease West Nile fever.

West Nile fever is a vector-borne zoonotic viral disease spread by mosquitoes and transferable between both humans and animals. The causative agent, West Nile virus (WNV), is endemic in large areas of Africa, the Middle East and West Asia. Until the mid-1990s, the disease was mainly linked to influenza-like illness with only occasional reports of meningitis and encephalitis. However, WNV was recognized as a major pathogen after an unexpected outbreak in New York City (USA) in 1999 and outbreaks in Romania and Israel. The change of epidemiology was accompanied by instances of severe meningitis, encephalitis and human fatalities. This increase in virulence and changing epidemiology led to increased interest in the development of WNV vaccines; however, there are no commercially available vaccines to date for humans against WNV. Isconova will lead a part of this EU-financed project aimed at optimizing a protein-based vaccine through the use of Isconova's adjuvant Matrix $M^{\mathrm{TM}}$.

"That we have been chosen for the WINGS-project is not only another testament to the strength of our product platform but also to our unique expertise of adjuvants and vaccine systems. The project is incredibly interesting for us as it will give us additional documentation for Matrix $M$ and may result in a very valuable vaccine, while being fully financed by a third party," said Isconova's CEO, Lena Söderström.

Sources: Isconova, Sweden: www.isconova.com 


\section{Antigen/adjuvant-carrying nanoparticles show promise for vaccine delivery}

A team of researchers from Massachusetts Institute of Technology (MIT; Cambridge, MA, USA) has demonstrated the efficacy of a novel nanoparticle vaccine vector, providing new possibilities for therapeutic protein delivery in infectious diseases such as HIV and malaria.

\begin{abstract}
"The potential of the new vesicles in vaccine delivery is promising but further work is necessary to demonstrate the utility of the particles in the prevention of human disease."
\end{abstract}

The authors of the study, published in Nature Materials, describe the development of interbilayer-cross-linked multilamellar vesicles (ICMVs), formed by cross-linking head groups of adjacent lipid bilayers within multilamellar vesicles. These lipid vesicles can carry protein antigens in the vesicle core and immunostimulatory drugs in the vesicle walls yet exhibit rapid release in the presence of endolysosomal lipases. The study found that these antigen/adjuvant-carrying vesicles serve as a highly effective vaccine, with low doses eliciting strong endogenous T-cell and antibody responses. Indeed, after immunization, up to $30 \%$ of all killer $\mathrm{T}$ cells in the mice were specific to the vaccine protein. "That is one of the strongest T-cell responses generated by a protein vaccine, and comparable to strong viral vaccines, but without the safety concerns of live viruses," said lead author Darrell Irvine, associate professor at MIT's Department of Biological Engineering.

Currently, the most effective method of stimulating both $\mathrm{T}$ - and $\mathrm{B}$-cell-mediated immune responses is to use inactivated or attenuated vaccines that contain dead or attenuated microorganisms. However, this is difficult to achieve with some viruses, such as HIV, owing to the difficulties of reducing the virulence of the virus. As an alternative, researchers have developed vaccines based on recombinant proteins. However, while the immunogenicity of synthetic vaccines avoid the toxicity and antivector immunity associated with live vaccine vectors, their is poor; in particular, they elicit only weak $\mathrm{CD} 8^{+}$ T-cell responses. Recently, researchers have designed vaccines enclosed in liposomes, which could help promote T-cell responses by packaging the protein in a virus-like particle. However, these vesicles are somewhat unstable in blood and body fluids, preventing them from stimulating an effective T-cell response. Irvine and colleagues elaborated on this design by packaging many of the droplets together in concentric spheres. Following fusion, the adjacent walls of the liposomes are "stapled" to each other, conferring stability and minimizing premature break-down following injection.

The potential of the new vesicles in vaccine delivery is promising but further work is necessary to demonstrate the utility of the particles in the prevention of human disease. Indeed, "Efforts are underway in our laboratory to translate these promising results into effective vaccines against malaria and HIV," said coauthor James Moon, also at the
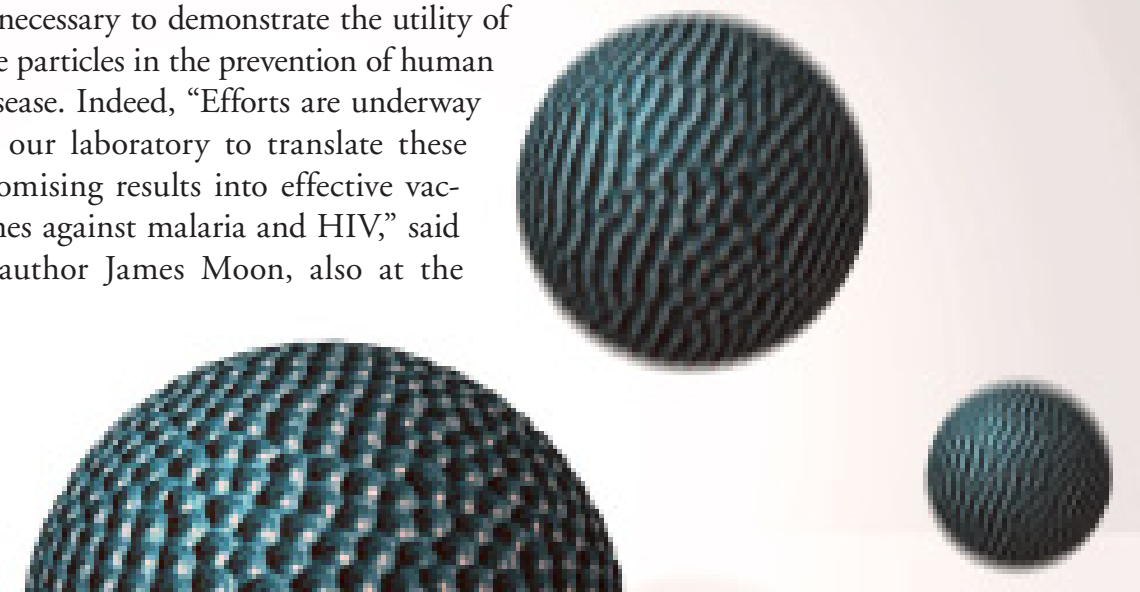

Department of Biological Engineering at MIT. "In collaboration with researchers at Walter Reed Army Institute of Research, we have thus far achieved very strong humoral responses against malaria parasites with ICMV vaccines carrying candidate malaria antigen, and we are pursuing to demonstrate their efficacy in non-human primate models. As for HIV, we are also working with other investigators at the Ragon Institute of MGH, MIT and Harvard to examine ICMVs as a potent HIV vaccine carrier."

Sources: Moon JJ, Suh $H$, Bershteyn $A$ et al.: Interbilayer-crosslinked multilamellar vesicles as synthetic vaccines for potent humoral and cellular immune responses. Nat. Mater. 10(3), 243-251 (2011); MIT news: http://web.mit.edu/newsoffice/2011/nano-sized-vaccines-0222.html 